\title{
Locally Lipschitz continuous integrated semigroups
}

\author{
by \\ NAOKI TANAKA (Okayama)
}

\begin{abstract}
This paper is concerned with the problem of real characterization of locally Lipschitz continuous $(n+1)$-times integrated semigroups, where $n$ is a nonnegative integer. It is shown that a linear operator is the generator of such an integrated semigroup if and only if it is closed, its resolvent set contains all sufficiently large real numbers, and a stability condition in the spirit of the finite difference approximation theory is satisfied.
\end{abstract}

1. Introduction. Let $X$ be a Banach space and $B(X)$ the set of all bounded linear operators from $X$ into itself. Let $n$ be a positive integer. A family $\{U(t) ; t \geq 0\}$ in $B(X)$ is called an $n$-times integrated semigroup on $X$ if the following conditions are satisfied:

(I1) $U(\cdot) x:[0, \infty) \rightarrow X$ is continuous for $x \in X$.

$$
\begin{aligned}
U(t) U(s) x=\frac{1}{(n-1) !}\left(\int_{t}^{t+s}(t+s-r)^{n-1} U(r) x d r\right. & \\
& \left.-\int_{0}^{s}(t+s-r)^{n-1} U(r) x d r\right)
\end{aligned}
$$

for $x \in X$ and $t, s \geq 0$.

(I3) $U(t) x=0$ for all $t>0$ implies $x=0$.

Let $\{U(t) ; t \geq 0\}$ be an $n$-times integrated semigroup on $X$. Then the generator $A$ of $\{U(t) ; t \geq 0\}$ is defined in the following way: $x \in D(A)$ and $y=A x$ if and only if

$$
U(t) x=\int_{0}^{t} U(r) y d r+\frac{t^{n}}{n !} x \quad \text { for } t \geq 0 .
$$

The densely defined generators in the exponentially bounded case were characterized by Neubrander [10], and his result was extended to the following two cases:

2000 Mathematics Subject Classification: Primary 47D62; Secondary 47D60. 
(i) Arendt [1] gave a characterization of the generator of an $(n+1)$-times integrated semigroup $\{U(t) ; t \geq 0\}$ on $X$ which is exponentially Lipschitz continuous in the sense that $\|U(t+h)-U(t)\| \leq M e^{\omega(t+h)} h$ for $t \geq 0$ and $h \geq 0$, in the case where the domain of the generator is not necessarily dense in $X$.

(ii) Tanaka and Okazawa [14] characterized the densely defined generators of $n$-times integrated semigroups which are not necessarily exponentially bounded.

An integrated semigroup $\{U(t) ; t \geq 0\}$ on $X$ is said to be locally Lipschitz continuous if for each $\tau>0$ there exists $L_{\tau}>0$ such that $\|U(t)-U(s)\| \leq$ $L_{\tau}|t-s|$ for $t, s \in[0, \tau]$. It is shown in the final part of Section 3 that there exists a non-densely defined operator which is the generator of a locally Lipschitz continuous twice integrated semigroup on $l^{\infty}$ but not the generator of any exponentially Lipschitz continuous $(n+1)$-times integrated semigroup on $l^{\infty}$ for every nonnegative integer $n$. The twice integrated semigroup constructed cannot be dealt with by the above-mentioned results.

We are interested in locally Lipschitz continuous $(n+1)$-times integrated semigroups which are not necessarily exponentially bounded and in characterizing their generators whose domains are not necessarily dense in $X$. If $n=0$ then our objective has already been accomplished, since Kellerman and Hieber [5] showed that every locally Lipschitz continuous once integrated semigroup is always exponentially Lipschitz continuous. The main result of this paper is given by

Main Theorem. Let $n$ be a nonnegative integer. An operator $A$ is the generator of a locally Lipschitz continuous $(n+1)$-times integrated semigroup $\{U(t) ; t \geq 0\}$ on $X$ if and only if the following conditions are satisfied:

(A1) $A$ is a closed linear operator in $X$ whose resolvent set contains $(\omega, \infty)$ for some $\omega \geq 0$.

(A2) For each $\tau>0$ there exists $M_{\tau}>0$ such that

$$
\left\|\prod_{l=1}^{k}\left(I-h_{l} A\right)^{-1} x\right\| \leq M_{\tau}\|x\|_{n}
$$

for $x \in D\left(A^{n}\right)$ and every finite sequence $\left\{h_{l}\right\}_{l=1}^{k}$ of positive numbers such that $h_{l} \omega<1$ for $1 \leq l \leq k$ and $\sum_{l=1}^{k} h_{l} \leq \tau$. Here $\|x\|_{n}=$ $\sum_{j=0}^{n}\left\|A^{j} x\right\|$ for $x \in D\left(A^{n}\right)$.

Condition (A2) can be regarded as a stability condition from the viewpoint of the finite difference approximation theory. In fact, if $\left\{0=t_{0}<t_{1}<\right.$ $\left.\cdots<t_{k} \leq \tau\right\}$ is a partition of $\left[0, t_{k}\right]$ with $\left(t_{l}-t_{l-1}\right) \omega<1$ for $1 \leq l \leq k$ and $\left\{x_{l}\right\}_{l=1}^{k}$ is a solution of the finite difference equation

$$
\left(x_{l}-x_{l-1}\right) /\left(t_{l}-t_{l-1}\right)=A x_{l} \quad \text { for } 1 \leq l \leq k
$$


with the initial condition $x_{0}=x \in D\left(A^{n}\right)$, then condition (A2) gives the estimate $\left\|x_{l}\right\| \leq M_{\tau}\|x\|_{n}$ for $1 \leq l \leq k$. For this reason, condition (A2) is natural in the study of the abstract Cauchy problem

$(\mathrm{ACP} ; x) \quad u^{\prime}(t)=A u(t) \quad$ for $t \geq 0, \quad u(0)=x$.

By a solution $u$ to $(\mathrm{ACP} ; x)$ we mean that $u \in C^{1}([0, \infty) ; X)$ and $u$ satisfies the equation $(\mathrm{ACP} ; x)$.

In Section 2 we investigate some basic properties of generators of locally Lipschitz continuous $(n+1)$-times integrated semigroups and prove the necessity part of the main theorem. Section 3 concerns the generation of locally Lipschitz continuous $(n+1)$-times integrated semigroups on $X$ and the relationship between the main theorem and some previous results.

\section{Basic properties of generators of locally Lipschitz continuous} integrated semigroups. Let $n$ be a nonnegative integer. Let $A$ be the generator of a locally Lipschitz continuous $(n+1)$-times integrated semigroup $\{U(t) ; t \geq 0\}$ on $X$. Then it is known [9] that $A$ is a closed linear operator in $X$ with the following properties:

$$
\begin{aligned}
& U(t) x \in D(A), \quad A U(t) x=U(t) A x \quad \text { for } x \in D(A) \text { and } t \geq 0, \\
& \int_{0}^{t} U(s) x d s \in D(A), \quad A \int_{0}^{t} U(s) x d s=U(t) x-\frac{t^{n+1}}{(n+1) !} x \\
& \text { for } x \in X \text { and } t \geq 0 .
\end{aligned}
$$

To investigate some properties of the generator of a locally Lipschitz continuous $(n+1)$-times integrated semigroup, we use a method similar to that due to Sanekata [12], but more delicate arguments are required here. For each $\lambda \in \mathbb{C}$ with $\operatorname{Re} \lambda>0$, define $R_{0}(\lambda) \in B(X)$ by

$$
R_{0}(\lambda) x=\lambda^{n+1} \int_{0}^{1} e^{-\lambda t} U(t) x d t \quad \text { for } x \in X .
$$

LEMma 2.1. There exists $M>0$ such that

$$
\left\|R_{0}(\lambda)\right\| \leq M|\lambda|^{n} \quad \text { for } \lambda \in \mathbb{C} \text { with } \operatorname{Re} \lambda>0 .
$$

Proof. Let $\lambda \in \mathbb{C}$ and $\operatorname{Re} \lambda>0$. Let $x \in X$ and $x^{*} \in X^{*}$. By the local Lipschitz continuity of $\{U(t) ; t \geq 0\}$, we see that $\left\langle x^{*}, U(t) x\right\rangle$ is Lipschitz continuous on $[0,1]$ and $\left|(d / d t)\left\langle x^{*}, U(t) x\right\rangle\right| \leq L\left\|x^{*}\right\|\|x\|$ for almost all $t \in$ $(0,1)$, where $L>0$ is a constant. By integration by parts we have

$$
\left\langle x^{*}, R_{0}(\lambda) x\right\rangle=\lambda^{n}\left(-e^{-\lambda}\left\langle x^{*}, U(1) x\right\rangle+\int_{0}^{1} e^{-\lambda t}(d / d t)\left\langle x^{*}, U(t) x\right\rangle d t\right) .
$$

The desired inequality follows readily from this equality. 
For each $\lambda \in \mathbb{C}$ with $\operatorname{Re} \lambda>0$, define $Q_{0}(\lambda) \in B(X)$ by

$$
Q_{0}(\lambda) x=\lambda^{n+1} e^{-\lambda} U(1) x+\sum_{k=0}^{n} e^{-\lambda} \frac{\lambda^{k}}{k !} x \quad \text { for } x \in X .
$$

Lemma 2.2. (i) There exists $M>0$ such that

$$
\left\|Q_{0}(\lambda)\right\| \leq M e^{-\operatorname{Re} \lambda}(1+|\lambda|)^{n+1} \quad \text { for } \lambda \in \mathbb{C} \text { with } \operatorname{Re} \lambda>0 .
$$

(ii) For each $\lambda \in \mathbb{C}$ with $\operatorname{Re} \lambda>0$ we have

$$
\begin{gathered}
R_{0}(\lambda)(\lambda I-A) x=\left(I-Q_{0}(\lambda)\right) x \quad \text { for } x \in D(A), \\
R_{0}(\lambda) x \in D(A), \quad(\lambda I-A) R_{0}(\lambda)=\left(I-Q_{0}(\lambda)\right) x \quad \text { for } x \in X .
\end{gathered}
$$

Proof. Let $\lambda \in \mathbb{C}$ and $\operatorname{Re} \lambda>0$. Since $\max _{0 \leq k \leq n}(1 / k !) \leq e$ and

$$
(1+|\lambda|)^{n+1} \geq \sum_{k=0}^{n+1}|\lambda|^{k},
$$

assertion (i) is easily verified. To prove (ii), let $x \in X$. Since

$$
R_{0}(\lambda) x=\lambda^{n+1}\left(e^{-\lambda} \int_{0}^{1} U(s) x d s+\int_{0}^{1} \lambda e^{-\lambda t}\left(\int_{0}^{t} U(s) x d s\right) d t\right)
$$

it follows from (2.2) that $R_{0}(\lambda) x \in D(A)$ and

$$
\begin{aligned}
(\lambda I-A) R_{0}(\lambda) x= & \frac{\lambda^{n+1}}{(n+1) !} e^{-\lambda} x+\int_{0}^{1} \lambda e^{-\lambda t} \frac{(\lambda t)^{n+1}}{(n+1) !} x d t \\
& -\lambda^{n+1} e^{-\lambda} U(1) x .
\end{aligned}
$$

Integration by parts yields

$$
\int_{0}^{1} \lambda e^{-\lambda t} \frac{(\lambda t)^{n+1}}{(n+1) !} d t=1-e^{-\lambda} \sum_{k=0}^{n+1} \frac{\lambda^{k}}{k !} .
$$

Substituting this equality into (2.5) we obtain (2.4). Since $A$ is closed, by (2.1) we have $R_{0}(\lambda) z \in D(A)$ and $A R_{0}(\lambda) z=R_{0}(\lambda) A z$ for $z \in D(A)$. This fact together with (2.4) implies (2.3).

Proposition 2.3. (i) The resolvent set of $A$ contains a region

$$
\begin{aligned}
\Omega= & \{\lambda \in \mathbb{C} \backslash \mathbb{R} ; \operatorname{Re} \lambda \geq \alpha \log |\operatorname{Im} \lambda|+\beta, \operatorname{Re} \lambda \geq \gamma\} \\
& \cup\{\lambda \in \mathbb{R} ; \lambda \geq \gamma\},
\end{aligned}
$$

where $\alpha, \beta$ and $\gamma$ are positive numbers.

(ii) There exists $M>0$ such that

$$
\left\|(\lambda I-A)^{-1}\right\| \leq M|\lambda|^{n} \quad \text { for } \lambda \in \Omega .
$$


Proof. Let $\xi \in \mathbb{R}$ and $\eta \in \mathbb{R}$ satisfy

$$
\eta \neq 0, \quad \xi \geq \alpha \log |\eta|+\beta, \quad \xi \geq \gamma
$$

where $\alpha, \beta$ and $\gamma$ are yet to be determined. Then we have $|\eta|^{\alpha} \leq e^{-\beta+\xi}$. If $\alpha>0$ is chosen such that $\alpha=n+1$, then $e^{-\xi}\left((1+|\xi|)^{n+1}+|\eta|^{n+1}\right)$ vanishes as $\xi \rightarrow \infty$ and $\beta \rightarrow \infty$. This fact implies that the positive numbers $\gamma$ and $\beta$ can be chosen so large that $\left\|Q_{0}(\lambda)\right\| \leq 1 / 2$ for $\lambda \in \Omega$, by Lemma 2.2(i). Since $\left(I-Q_{0}(\lambda)\right)^{-1} \in B(X)$ exists and $\left\|\left(I-Q_{0}(\lambda)\right)^{-1}\right\| \leq 2$ for $\lambda \in \Omega$, the proposition follows from Lemmas 2.1 and 2.2.

To prove the necessity part of the main theorem, we define

$$
T_{h}(t) x=h^{-1}(U(t+h)-U(t)) A^{n} x+\sum_{k=0}^{n-1} \frac{t^{k}}{k !} A^{k} x
$$

for $x \in D\left(A^{n}\right), t \geq 0$ and $h \neq 0$, and

$$
\varepsilon_{h}(t)=\frac{t^{n}}{n !}-\frac{1}{h}\left(\frac{(t+h)^{n+1}}{(n+1) !}-\frac{t^{n+1}}{(n+1) !}\right)
$$

for $t \geq 0$ and $h \neq 0$.

Lemma 2.4. Let $x \in D\left(A^{n}\right)$ and $\lambda \in \Omega$. Then

$$
\begin{aligned}
(\lambda I-A)^{-1} x= & \int_{0}^{\tau_{0}} e^{-\lambda t} T_{h}(t) x d t+e^{-\lambda \tau_{0}} T_{h}\left(\tau_{0}\right)(\lambda I-A)^{-1} x \\
& +\left(e^{-\lambda \tau_{0}} \varepsilon_{h}\left(\tau_{0}\right)+\int_{0}^{\tau_{0}} \lambda e^{-\lambda t} \varepsilon_{h}(t) d t\right)(\lambda I-A)^{-1} A^{n} x \\
& -h^{-1} \int_{0}^{h} U(s) A^{n+1}(\lambda I-A)^{-1} x d s
\end{aligned}
$$

for any $\tau_{0}>0$ and $h \neq 0$.

Proof. Let $x \in D\left(A^{n}\right), \lambda \in \Omega, \tau_{0}>0$ and $h \neq 0$. Integration by parts yields

$$
\begin{aligned}
\int_{0}^{\tau_{0}} e^{-\lambda t}(U(t+h)-U(t)) & A^{n} x d t \\
= & e^{-\lambda \tau_{0}} \int_{0}^{\tau_{0}}(U(s+h)-U(s)) A^{n} x d s \\
& +\int_{0}^{\tau_{0}} \lambda e^{-\lambda t}\left(\int_{0}^{t}(U(s+h)-U(s)) A^{n} x d s\right) d t .
\end{aligned}
$$


By (2.2) we see that the right-hand side belongs to $D(A)$ and

$$
\begin{aligned}
& A \int_{0}^{\tau_{0}} e^{-\lambda t}(U(t+h)-U(t)) A^{n} x d t \\
& =e^{-\lambda \tau_{0}}\left(U\left(\tau_{0}+h\right) A^{n} x-\frac{\left(\tau_{0}+h\right)^{n+1}}{(n+1) !} A^{n} x-U\left(\tau_{0}\right) A^{n} x+\frac{\tau_{0}^{n+1}}{(n+1) !} A^{n} x\right) \\
& \quad+\int_{0}^{\tau_{0}} \lambda e^{-\lambda t}\left(U(t+h) A^{n} x-\frac{(t+h)^{n+1}}{(n+1) !} A^{n} x\right. \\
& \left.\quad-U(t) A^{n} x+\frac{t^{n+1}}{(n+1) !} A^{n} x\right) d t \\
& \quad\left(U(h) A^{n} x-\frac{h^{n+1}}{(n+1) !} A^{n} x\right) .
\end{aligned}
$$

The last term on the right-hand side is equal to $-A \int_{0}^{h} U(s) A^{n} x d s$ by (2.2). By integration by parts we have

$$
A \int_{0}^{\tau_{0}} e^{-\lambda t} \sum_{k=0}^{n-1} \frac{t^{k}}{k !} A^{k} x d t=e^{-\lambda \tau_{0}} \sum_{k=1}^{n} \frac{\left(\tau_{0} A\right)^{k}}{k !} x+\lambda \int_{0}^{\tau_{0}} e^{-\lambda t} \sum_{k=1}^{n} \frac{(t A)^{k}}{k !} x d t .
$$

The desired equality is obtained by combining the equalities above.

Let $\tau>0$ and choose $\tau_{0}>2 \tau$. By the local Lipschitz continuity of $\{U(t) ; t \geq 0\}$ there exists $M_{\tau}>0$ such that $\left\|T_{h}(t) x\right\| \leq M_{\tau}\|x\|_{n}$ for $t \in\left[0, \tau_{0}\right]$ and $h \in(0,1]$. Here and below $M_{\tau}$ denotes various constants depending on $\tau$.

Let $\alpha, \beta$ and $\gamma$ be the positive numbers in Proposition 2.3(i), and let $\alpha_{0}>\max \left\{\alpha,(n+1) /\left(\tau_{0}-2 \tau\right)\right\}, \beta_{0}>\beta, \omega_{0}>\max \left\{\gamma, \beta_{0}\right\}$ and $\eta_{0}=\exp \left(\left(\omega_{0}-\right.\right.$ $\left.\left.\beta_{0}\right) / \alpha_{0}\right)$. Then we define $\Gamma=\Gamma_{1} \cup \Gamma_{2}$, where $\Gamma_{1}=\left\{\zeta=\xi+i \eta ; \xi=\alpha_{0} \log |\eta|\right.$ $\left.+\beta_{0},|\eta| \geq \eta_{0}\right\}$ and $\Gamma_{2}=\left\{\zeta=\omega_{0}+i \eta ;|\eta| \leq \eta_{0}\right\}$. Here $i$ stands for the imaginary unit. Notice that $\Gamma$ is oriented so that $\operatorname{Im} \zeta$ increases along $\Gamma$.

Lemma 2.5. Let $x^{*} \in X^{*}$ and $x \in D\left(A^{n}\right)$. Then there exist a measurable function $f$ on $\left(0, \tau_{0}\right)$ and a holomorphic function $g$ on $\Omega$ such that

$$
\begin{gathered}
|f(t)| \leq M_{\tau}\left\|x^{*}\right\|\|x\|_{n} \quad \text { for almost all } t \in\left(0, \tau_{0}\right), \\
|g(\zeta)| \leq M_{\tau}|\zeta|^{n}\left\|x^{*}\right\|\|x\|_{n} \quad \text { for } \zeta \in \Omega, \\
\left\langle x^{*},(\lambda I-A)^{-1} x\right\rangle=\int_{0}^{\tau_{0}} e^{-\lambda t} f(t) d t+\frac{1}{2 \pi i} \int_{\Gamma} \frac{1}{\lambda-\zeta} e^{-\zeta \tau_{0}} g(\zeta) d \zeta \\
\quad \text { for } \lambda>\omega_{0} .
\end{gathered}
$$

Proof. Let $x^{*} \in X^{*}$ and $x \in D\left(A^{n}\right)$. Since $\left\langle x^{*}, U(t) A^{n} x\right\rangle$ is Lipschitz continuous on $\left[0, \tau_{0}\right]$, it is differentiable for almost all $t \in\left(0, \tau_{0}\right)$, so that the limit $f(t):=\lim _{h \downarrow 0}\left\langle x^{*}, T_{h}(t) x\right\rangle$ exists for almost all $t \in\left(0, \tau_{0}\right)$. Clearly, 
$f$ is measurable and satisfies (2.7). If $\alpha_{0} \tau_{0}>n+1$ and $\lambda>\omega_{0}$ then $\int_{\Gamma}|\lambda-\zeta|^{-1} e^{-(\operatorname{Re} \zeta) \tau_{0}}|\zeta|^{n}|d \zeta|<\infty$ and the integral

$$
\int_{R-s_{R} i}^{R+s_{R} i}|\lambda-\zeta|^{-1} e^{-(\operatorname{Re} \zeta) \tau_{0}}|\zeta|^{n}|d \zeta|
$$

tends to zero as $R \rightarrow \infty$, where $s_{R}=\exp \left(\left(R-\beta_{0}\right) / \alpha_{0}\right)$. Since $\Gamma \subset \Omega$, by Cauchy's integral formula we have

$$
e^{-\lambda \tau_{0}} T_{h}\left(\tau_{0}\right)(\lambda I-A)^{-1} x=\frac{1}{2 \pi i} \int_{\Gamma} \frac{1}{\lambda-\zeta} e^{-\zeta \tau_{0}} T_{h}\left(\tau_{0}\right)(\zeta I-A)^{-1} x d \zeta
$$

for $\lambda>\omega_{0}$ and $h \in(0,1]$. Here we have used Proposition 2.3(ii) and the condition $\alpha_{0} \tau_{0}>n+1$. Since $U(\cdot) z:[0, \infty) \rightarrow X$ is differentiable for $z \in D(A)$, the limit $g(\zeta):=\lim _{h \downarrow 0}\left\langle x^{*}, T_{h}\left(\tau_{0}\right)(\zeta I-A)^{-1} x\right\rangle$ exists for all $\zeta \in \Omega$. By Proposition 2.3(ii) we see that $g$ satisfies (2.8). Taking the weak limit in (2.6) as $h \downarrow 0$ we obtain (2.9) by Lebesgue's convergence theorem. Here we have used the strong continuity of $\{U(t) ; t \geq 0\}$ and $U(0)=0$.

Set $R(\lambda)=(\lambda I-A)^{-1}$ for $\lambda \in \Omega$. By applying the resolvent equation $(\lambda-\mu) R(\lambda) R(\mu)=R(\mu)-R(\lambda)$ for $\lambda, \mu \in \Omega$, the following lemma can be proved by induction.

Lemma 2.6. Let $k \geq 2$. Then

$$
\prod_{l=1}^{k} R\left(\lambda_{l}\right)=(-1)^{k-1} \int_{D_{k-1}} F_{\lambda_{k}, \ldots, \lambda_{1}}\left(\sigma_{1}, \ldots, \sigma_{k-1}\right) d \sigma_{1} \cdots d \sigma_{k-1}
$$

for every finite sequence $\left\{\lambda_{l}\right\}_{l=1}^{k}$ with $\lambda_{l}>\omega_{0}$ for $1 \leq l \leq k$, where

$$
\begin{array}{r}
F_{\lambda_{k}, \ldots, \lambda_{1}}\left(\sigma_{1}, \ldots, \sigma_{k-1}\right) \\
=R^{(k-1)}\left(\lambda_{1} \sigma_{1}+\cdots+\lambda_{k-1} \sigma_{k-1}+\lambda_{k}\left(1-\sum_{l=1}^{k-1} \sigma_{l}\right)\right), \\
D_{k-1}=\left\{\left(\sigma_{1}, \ldots, \sigma_{k-1}\right) ; \sigma_{l} \geq 0 \text { for } 1 \leq l \leq k-1 \text { and } \sum_{l=1}^{k-1} \sigma_{l} \leq 1\right\} .
\end{array}
$$

Lemma 2.7. For $x \in D\left(A^{n}\right)$ and every finite sequence $\left\{h_{l}\right\}_{l=1}^{k}$ of positive numbers with $h_{l} \omega_{0}<1$ for $1 \leq l \leq k$ and $k \geq 1$, we have

$$
\begin{aligned}
& \left\|\prod_{l=1}^{k}\left(I-h_{l} A\right)^{-1} x\right\| \\
& \quad \leq M_{\tau}\left(1+\frac{1}{2 \pi} \int \prod_{\Gamma}\left|1-\zeta h_{l}\right|^{-1} e^{-(\operatorname{Re} \zeta) \tau_{0}}|\zeta|^{n}|d \zeta|\right)\|x\|_{n} .
\end{aligned}
$$


Proof. Let $x \in D\left(A^{n}\right)$ and $x^{*} \in X^{*}$. Let $k \geq 2$ and let $\left\{\lambda_{l}\right\}_{l=1}^{k}$ be any sequence such that $\lambda_{l}>\omega_{0}$ for $1 \leq l \leq k$. If $\left(\sigma_{1}, \ldots, \sigma_{k-1}\right) \in D_{k-1}$ then $\lambda_{1} \sigma_{1}+\cdots+\lambda_{k-1} \sigma_{k-1}+\lambda_{k}\left(1-\sum_{l=1}^{k-1} \sigma_{l}\right)>\omega_{0}$. By (2.9) and (2.10) we see that $\left\langle x^{*}, \prod_{l=1}^{k} R\left(\lambda_{l}\right) x\right\rangle$ can be written as

$\int_{D_{k-1}} d \sigma_{1} \cdots d \sigma_{k-1}\left(\int_{0}^{\tau_{0}} t^{k-1} e^{-\left(\lambda_{1} \sigma_{1}+\cdots+\lambda_{k-1} \sigma_{k-1}+\lambda_{k}\left(1-\sum_{l=1}^{k-1} \sigma_{l}\right)\right) t} f(t) d t\right.$

$\left.+\frac{1}{2 \pi i} \int_{\Gamma}(-1)^{k-1} r_{\zeta}^{(k-1)}\left(\lambda_{1} \sigma_{1}+\cdots+\lambda_{k-1} \sigma_{k-1}+\lambda_{k}\left(1-\sum_{l=1}^{k-1} \sigma_{l}\right)\right) e^{-\zeta \tau_{0}} g(\zeta) d \zeta\right)$,

where $r_{\zeta}(\lambda)=(\lambda-\zeta)^{-1}$ for $\lambda>\omega_{0}$ and $\zeta \in \Gamma$. Changing the variable, by Lemma 2.6 we have

$$
\begin{array}{r}
\int_{D_{k-1}} d \sigma_{1} \cdots d \sigma_{k-1} \int_{0}^{\infty} t^{k-1} e^{-\left(\lambda_{1} \sigma_{1}+\cdots+\lambda_{k-1} \sigma_{k-1}+\lambda_{k}\left(1-\sum_{l=1}^{k-1} \sigma_{l}\right)\right) t} d t \\
=\left(\lambda_{1} \cdots \lambda_{k}\right)^{-1}
\end{array}
$$

Since

$$
\begin{aligned}
& \int_{\Gamma}\left|r_{\zeta}^{(k-1)}\left(\lambda_{1} \sigma_{1}+\cdots+\lambda_{k-1} \sigma_{k-1}+\lambda_{k}\left(1-\sigma_{1}-\cdots-\sigma_{k-1}\right)\right) e^{-\zeta \tau_{0}} g(\zeta)\right||d \zeta| \\
& \quad \leq\left((-1)^{k-1} r_{\omega_{0}}^{(k-1)}\left(\lambda_{1} \sigma_{1}+\cdots+\lambda_{k-1} \sigma_{k-1}+\lambda_{k}\left(1-\sigma_{1}-\cdots-\sigma_{k-1}\right)\right)+1\right) \\
& \quad \times M_{\tau}\left\|x^{*}\right\|\|x\|_{n}
\end{aligned}
$$

and the right-hand side is integrable on $D_{k-1}$, we apply Fubini's theorem to find

$$
\begin{aligned}
(-1)^{k-1} \int_{D_{k-1}} d \sigma_{1} \cdots d \sigma_{k-1} \int_{\Gamma} r_{\zeta}^{(k-1)}\left(\sum_{l=1}^{k-1} \lambda_{l} \sigma_{l}\right. & \left.+\lambda_{k}\left(1-\sum_{l=1}^{k-1} \sigma_{l}\right)\right) e^{-\zeta \tau_{0}} g(\zeta) d \zeta \\
& =\int_{\Gamma} \prod_{l=1}^{k}\left(\lambda_{l}-\zeta\right)^{-1} e^{-\zeta \tau_{0}} g(\zeta) d \zeta
\end{aligned}
$$

The desired inequality is obtained by combining these equalities.

Once the following lemma is shown, the proof of the necessity part of the main theorem is completed, since the right-hand side of $(2.11)$ is bounded by $M_{\tau}\|x\|_{n}$, under the condition $\alpha_{0}>(n+1) /\left(\tau_{0}-2 \tau\right)$.

Lemma 2.8. There exists $M_{\tau}>0$ such that

$$
\prod_{l=1}^{k}\left|1-\zeta h_{l}\right|^{-1} \leq M_{\tau} \exp (2 \tau \operatorname{Re} \zeta)
$$

for $\zeta \in \Gamma$ and every finite sequence $\left\{h_{l}\right\}_{l=1}^{k}$ of positive numbers with $h_{l} \omega_{0} \leq$ $1 / 2$ for $1 \leq l \leq k$ and $\sum_{l=1}^{k} h_{l} \leq \tau$. 
Proof. Let $\zeta=\xi+i \eta \in \Gamma$ and let $\left\{h_{l}\right\}_{l=1}^{k}$ be a finite sequence of positive numbers such that $h_{l} \omega_{0} \leq 1 / 2$ for $1 \leq l \leq k$ and $\sum_{l=1}^{k} h_{l} \leq \tau$. We divide the set $\{1, \ldots, k\}$ into the disjoint sets $I_{1}=\left\{l ; h_{l} \xi \in[0,1 / 2]\right\}$ and $I_{2}=$ $\left\{l ; \zeta=\xi+i \eta \in \Gamma_{1}, h_{l} \xi>1 / 2\right\}$. If $l \in I_{1}$ then

$$
\left|1-\zeta h_{l}\right|^{-1} \leq\left(1-(\operatorname{Re} \zeta) h_{l}\right)^{-1} \leq \exp \left(2(\operatorname{Re} \zeta) h_{l}\right) .
$$

Here we have used the fact $(1-t)^{-1} \leq \exp (2 t)$ for $t \in[0,1 / 2]$. If $l \in I_{2}$ then

$$
\left|1-\zeta h_{l}\right|^{-1} \leq|\eta|^{-1} h_{l}^{-1} \leq 2 \xi /|\eta|
$$

Hence

$$
\prod_{l=1}^{k}\left|1-\zeta h_{l}\right|^{-1} \leq \exp (2 \xi \tau) \sup \left\{\left(2\left(\alpha_{0} \log |\eta|+\beta_{0}\right) /|\eta|\right)^{\left|I_{2}\right|} ;|\eta| \geq \eta_{0}\right\},
$$

where $\left|I_{2}\right|$ denotes the number of elements in $I_{2}$. To estimate $\left|I_{2}\right|$, let $\xi+i \eta$ $\in \Gamma_{1}$ and $h_{l} \xi>1 / 2$. Since $\xi=\alpha_{0} \log |\eta|+\beta_{0}>0$ we have

$$
\left|I_{2}\right| / 2=\sum_{l \in I_{2}}(1 / 2)<\xi \sum_{l \in I_{2}} h_{l} \leq\left(\alpha_{0} \log |\eta|+\beta_{0}\right) \tau,
$$

so that $\left|I_{2}\right| \leq 2\left(\alpha_{0} \log |\eta|+\beta_{0}\right) \tau$. Since $\lim _{t \rightarrow \infty}\left(\alpha_{0} \log t+\beta_{0}\right) / t=0$, it follows that the set $\left\{\eta ; 2\left(\alpha_{0} \log |\eta|+\beta_{0}\right) /|\eta|>1\right\}$ is bounded in $\mathbb{R}$. These facts together imply that $\left(2\left(\alpha_{0} \log |\eta|+\beta_{0}\right) /|\eta|\right)^{\left|I_{2}\right|}$ is bounded from above for $|\eta| \geq \eta_{0}$. The proof is thus complete.

\section{Generation of locally Lipschitz continuous integrated semi-} groups. Let $A$ be an operator in $X$ satisfying conditions (A1) and (A2). For simplicity of notation, we write $J_{h}=(I-h A)^{-1}$ for $h \in\left(0, h_{0}\right]$, where $h_{0}>0$ is such that $h_{0} \omega<1$. Let $\lambda, \mu \in\left(0, h_{0}\right]$ and set $A_{k, l}=J_{\lambda}^{k}-J_{\mu}^{l}$ for $k, l \geq 0$. To prove the sufficiency part of the main theorem, let $\tau>0$ and $z \in D\left(A^{n+1}\right)$. For $0 \leq k \leq[\tau / \lambda]$ and $0 \leq l \leq[\tau / \mu]$ we define

$$
a_{k, l}=\max _{p}\left\|J_{\sigma}^{p} A_{k, l} z\right\|,
$$

where $\sigma=\lambda \mu /(\lambda+\mu)$ and the maximum is taken over all nonnegative integers $p$ such that $\sigma p+\lambda k+\mu l \leq 2 \tau$. The use of the quantity $a_{k, l}$ is a new idea.

Lemma 3.1. For $0 \leq k \leq[\tau / \lambda]$ and $0 \leq l \leq[\tau / \mu]$ we have

$$
a_{k, l} \leq M_{2 \tau}\left((k \lambda-l \mu)^{2}+k \lambda^{2}+l \mu^{2}\right)^{1 / 2}\|A z\|_{n} .
$$

Proof. Let $0 \leq k \leq[\tau / \lambda]$ and consider any nonnegative integer $p$ such that $\sigma p+k \lambda \leq 2 \tau$. Since

$$
J_{\sigma}^{p} A_{k, 0} z=J_{\sigma}^{p} \sum_{j=1}^{k}\left(J_{\lambda}^{j} z-J_{\lambda}^{j-1} z\right)=\lambda \sum_{j=1}^{k} J_{\sigma}^{p} J_{\lambda}^{j} A z
$$


and $\left\|J_{\sigma}^{p} J_{\lambda}^{j} A z\right\| \leq M_{2 \tau}\|A z\|_{n}$ for $1 \leq j \leq k$ (by condition (A2)), we have $a_{k, 0} \leq M_{2 \tau}\|A z\|_{n} k \lambda$. Similarly, $a_{0, l} \leq M_{2 \tau}\|A z\|_{n} l \mu$.

Now, let $1 \leq k \leq[\tau / \lambda]$ and $1 \leq l \leq[\tau / \mu]$, and consider any positive integer $p$ with $p \sigma+k \lambda+l \mu \leq 2 \tau$. By the resolvent equation we have

$$
\begin{aligned}
& J_{\lambda} v=J_{\sigma}\left(\frac{\mu}{\lambda+\mu} v+\frac{\lambda}{\lambda+\mu} J_{\lambda} v\right), \\
& J_{\mu} w=J_{\sigma}\left(\frac{\lambda}{\lambda+\mu} w+\frac{\mu}{\lambda+\mu} J_{\mu} w\right) \quad \text { for } v, w \in X .
\end{aligned}
$$

Using these equalities with $v=J_{\lambda}^{k-1} z$ and $w=J_{\mu}^{l-1} z$ we find

$$
J_{\sigma}^{p} A_{k, l} z=\frac{\lambda}{\lambda+\mu} J_{\sigma}^{p+1} A_{k, l-1} z+\frac{\mu}{\lambda+\mu} J_{\sigma}^{p+1} A_{k-1, l} z .
$$

Since $\sigma \leq \lambda$ and $\sigma \leq \mu$, we notice that $(p+1) \sigma+k \lambda+(l-1) \mu \leq 2 \tau$ and $(p+1) \sigma+(k-1) \lambda+l \mu \leq 2 \tau$. By the definition of $a_{k, l-1}$ and $a_{k-1, l}$ we have

$$
a_{k, l} \leq \frac{\lambda}{\lambda+\mu} a_{k, l-1}+\frac{\mu}{\lambda+\mu} a_{k-1, l} .
$$

The desired inequality is proved by induction. (See also [7] and [16, Chapter XIV, Section 7].)

Proof of the sufficiency part of the main theorem. Let $x \in X$ and $\tau>0$. Choose $c>\omega$ and set $C=(c I-A)^{-(n+1)}$. By Lemma 3.1 we have

$$
\begin{aligned}
\| J_{\lambda}^{[t / \lambda]} C x & -J_{\mu}^{[s / \mu]} C x \| \\
& \leq M_{2 \tau}\left(([t / \lambda] \lambda-[s / \mu] \mu)^{2}+[t / \lambda] \lambda^{2}+[s / \mu] \mu^{2}\right)^{1 / 2}\|A C x\|_{n}
\end{aligned}
$$

for $\lambda, \mu \in\left(0, h_{0}\right]$ and $t \in[0, \tau]$. This implies that $S(t) x=\lim _{\lambda \downarrow 0} J_{\lambda}^{[t / \lambda]} C x$ exists for all $t \geq 0$ and the family $\{S(t) ; t \geq 0\}$ is a locally Lipschitz continuous $C$-regularized semigroup on $X$.

Let $z \in X$ and $t \geq 0$. Since

$$
\begin{aligned}
J_{\lambda}^{[t / \lambda]} C z-C z & =\sum_{k=1}^{[t / \lambda]}\left(J_{\lambda}^{k} C z-J_{\lambda}^{k-1} C z\right)=A\left(\lambda \sum_{k=1}^{[t / \lambda]} J_{\lambda}^{k} C z\right) \\
& =A \int_{\lambda}^{([t / \lambda]+1) \lambda} J_{\lambda}^{[s / \lambda]} C z d s,
\end{aligned}
$$

by the closedness of $A$ we have $\int_{0}^{t} S(s) z d s \in D(A)$ and

$$
A \int_{0}^{t} S(s) z d s=S(t) z-C z .
$$


For $k=1, \ldots, n+1$, define a family $\left\{V_{k}(t) ; t \geq 0\right\}$ in $B(X)$ by

$$
V_{k}(t) z=\int_{0}^{t} \int_{0}^{t_{1}} \cdots \int_{0}^{t_{k-1}} S\left(t_{k}\right) z d t_{k} \cdots d t_{1}
$$

for $z \in X$ and $t \geq 0$. Then it is proved similarly to [13, Lemma] that the following hold for $k=1, \ldots, n+1$.

(i) $V_{k}(t) z \in D\left(A^{k}\right)$ and $\int_{0}^{t}(c I-A)^{k-1} V_{k-1}(s) z d s \in D(A)$ for $z \in X$ and $t \geq 0$.

(ii) $(c I-A)^{k} V_{k}(t) \in B(X)$ and there exist $K_{k}>0$ and $\omega_{k} \geq 0$ such that

$$
\left\|(c I-A){ }^{k} V_{k}(t)-(c I-A)^{k} V_{k}(s)\right\| \leq \max \left(M_{2 \tau}, 1\right) K_{k} e^{\omega_{k} t}(t-s)
$$

for $0 \leq s \leq t \leq \tau$ and $\tau>0$.

(iii) We have

$$
\begin{aligned}
(c I-A)^{k} V_{k}(t)= & c(c I-A)^{k-1} V_{k}(t)-(c I-A)^{k-1} V_{k-1}(t) \\
& +\frac{t^{k-1}}{(k-1) !}(c I-A)^{k-1} C \quad \text { for } t \geq 0 .
\end{aligned}
$$

By the above fact with $k=n+1$, the family $\{U(t) ; t \geq 0\}$ in $B(X)$ defined by $U(t)=(c I-A)^{n+1} V_{n+1}(t)$ for $t \geq 0$ is locally Lipschitz continuous. By [14, Lemma 4.8] we see that $\{U(t) ; t \geq 0\}$ is an $(n+1)$-times integrated semigroup on $X$.

To prove that $A$ is the generator of $\{U(t) ; t \geq 0\}$, let $u \in D(A)$. The $(n+1)$-fold integration of (3.1) implies

$$
U(t) u=\frac{t^{n+1}}{(n+1) !} u+\int_{0}^{t} U(s) A u d s
$$

for $t \geq 0$. Hence $A \subset \mathfrak{A}$, where $\mathfrak{A}$ denotes the generator of $\{U(t) ; t \geq 0\}$. Since the intersection of the resolvent sets of $A$ and $\mathfrak{A}$ is nonempty, we have $A=\mathfrak{A}$.

The following asserts that a densely defined operator in $X$ is the generator of an $n$-times integrated semigroup on $X$ if and only if it satisfies conditions (A1) and (A2) of this paper.

COROLlary 1. Let n be a nonnegative integer. Let A be a densely defined linear operator in $X$. Then the following statements are mutually equivalent.

(i) $A$ is the generator of an n-times integrated semigroup on $X$.

(ii) $A$ is closed and the resolvent set $\varrho(A)$ of $A$ contains $(\omega, \infty)$ for some $\omega \geq 0$. For each $\tau>0$ there exists $M_{\tau}>0$ such that 


$$
\left\|\prod_{l=1}^{k}\left(I-h_{l} A\right)^{-1} x\right\| \leq M_{\tau}\|x\|_{n}
$$

for $x \in D\left(A^{n}\right)$ and every finite sequence $\left\{h_{l}\right\}_{l=1}^{k}$ of positive numbers with $h_{l} \omega<1$ for $1 \leq l \leq k$ and $\sum_{l=1}^{k} h_{l} \leq \tau$.

(iii) $A$ is closed and $\varrho(A) \supset(\beta, \infty)$ for some $\beta \geq 0$. For each $\tau>0$ there exists $K_{\tau}>0$ such that

$$
\sup \left\{\left\|\lambda^{k}(\lambda I-A)^{-k} x\right\| ; 0 \leq k / \lambda \leq \tau, \lambda>\beta, k \geq 1\right\} \leq K_{\tau}\|x\|_{n}
$$

for $x \in D\left(A^{n}\right)$.

(iv) $A$ is closed and $\varrho(A) \neq \emptyset$. The problem $(\mathrm{ACP} ; x)$ has a unique solution for each $x \in D\left(A^{n+1}\right)$.

Proof. If $A$ is the generator of an $n$-times integrated semigroup $\{U(t)$; $t \geq 0\}$ on $X$, then it is also the generator of the locally Lipschitz continuous $(n+1)$-times integrated semigroup $\{V(t) ; t \geq 0\}$ on $X$ defined by $V(t) x=\int_{0}^{t} U(s) x d s$ for $x \in X$ and $t \geq 0$. We therefore deduce from the Main Theorem that (i) implies (ii). The implication (ii) $\Rightarrow$ (iii) is obvious. It was proved by Oharu [11] that (iii) implies (iv). The implication (iv) $\Rightarrow$ (i) was shown in [9, Theorem 3.3].

We next deduce the Arendt theorem from the Main Theorem (although Arendt's original proof is quite elegant).

Corollary 2. Let $n$ be a nonnegative integer. Then $A$ is the generator of an exponentially Lipschitz continuous $(n+1)$-times integrated semigroup on $X$ if and only if it is a closed linear operator in $X$ and there exist $M>0$ and $a \geq 0$ such that $\varrho(A) \supset(a, \infty)$ and

$$
\left\|(1 /(k-1) !)(d / d \lambda)^{k-1}\left((\lambda I-A)^{-1} / \lambda^{n}\right)\right\| \leq M(\lambda-a)^{-k}
$$

for $\lambda>a$ and $k \geq 1$.

Proof. The necessity part is straightforward. We prove the sufficiency part using the Main Theorem. Since $A(\lambda I-A)^{-1}=\lambda(\lambda I-A)^{-1}-I$ for $\lambda>a$, it is shown inductively that

$$
A^{n}(\lambda I-A)^{-1} x=\lambda^{n}(\lambda I-A)^{-1} x-\sum_{l=0}^{n-1} \lambda^{l} A^{n-1-l} x
$$

for $x \in D\left(A^{n}\right)$ and $\lambda>a$. Dividing this equality by $\lambda^{n}$ and differentiating the resulting equality $k-1$ times, we find

$$
\begin{aligned}
\left\|(\lambda I-A)^{-k} x\right\| \leq & \frac{1}{(k-1) !}\left\|(d / d \lambda)^{k-1}\left((\lambda I-A)^{-1} / \lambda^{n}\right) A^{n} x\right\| \\
& +\lambda^{-k} \sum_{l=0}^{n-1}\left(\begin{array}{c}
n-l+k-2 \\
n-l-1
\end{array}\right) \lambda^{-(n-l-1)}\left\|A^{n-l-1} x\right\|
\end{aligned}
$$


for $x \in D\left(A^{n}\right), \lambda>a$ and $k \geq 1$. By (3.3) the first term on the righthand side is estimated by $M(\lambda-a)^{-k}\left\|A^{n} x\right\|$ for $\lambda>a$. Since $(1-t)^{-k}=$ $\sum_{p=0}^{\infty}\left(\begin{array}{c}k+p-1 \\ p\end{array}\right) t^{p}$ for $|t|<1$ and $k \geq 0$, the second term is bounded by $\lambda^{-k}(1-1 / \lambda)^{-k} \max _{0 \leq p \leq n-1}\left\|A^{p} x\right\|$ for $\lambda>\max (a, 1)$. Let $\beta=\max (a, 1)$ and $K=\max (M, 1)$. Then we have $(\beta, \infty) \subset \varrho(A)$ and $\left\|(\lambda I-A)^{-k} x\right\| \leq$ $K(\lambda-\beta)^{-k}\|x\|_{n}$ for $x \in D\left(A^{n}\right), \lambda>\beta$ and $k \geq 1$.

By an argument similar to that in $[8$, Section 4$]$ there exists a norm $N(\cdot)$ on the Banach space $D\left(A^{n}\right)$ equipped with the norm $\|\cdot\|_{n}$ such that $\|x\| \leq N(x) \leq K\|x\|_{n}$ for $x \in D\left(A^{n}\right)$ and $N\left((\lambda I-A)^{-1} x\right) \leq(\lambda-\beta)^{-1} N(x)$ for $x \in D\left(A^{n}\right)$ and $\lambda>\beta$. This fact shows that

$$
\left\|\prod_{l=1}^{k}\left(I-h_{l} A\right)^{-1} x\right\| \leq K \prod_{l=1}^{k}\left(1-h_{l} \beta\right)^{-1}\|x\|_{n}
$$

for $x \in D\left(A^{n}\right)$ and every finite sequence $\left\{h_{l}\right\}_{l=1}^{k}$ of positive numbers with $h_{l} \beta<1$ for $1 \leq l \leq k$. Since $(1-t)^{-1} \leq \exp (2 t)$ for $0 \leq t \leq 1 / 2$, we see that condition (A2) is satisfied with $M_{\tau}=K \exp (2 \beta \tau)$ and $\omega=2 \beta$. By the Main Theorem together with (3.2), $A$ is the generator of an $(n+1)$-times integrated semigroup $\{U(t) ; t \geq 0\}$ on $X$ and $\|U(t)-U(s)\| \leq \max \left(M_{2 \tau}, 1\right) L e^{b t}(t-s)$ for $0 \leq s \leq t \leq \tau$ and $\tau>0$. This means that $A$ is the generator of an exponentially Lipschitz continuous $(n+1)$-times integrated semigroup on $X$.

ExAmple. Let $X=l^{\infty}$. Let $\left(a_{k}\right)$ be the sequence in $\mathbb{C}$ defined by $a_{k}=$ $k+i e^{k^{2}}$ for $k \geq 1$, and define a linear operator $A$ in $X$ by $D(A)=\{x=$ $\left.\left(x_{k}\right) \in X ;\left(a_{k} x_{k}\right) \in X\right\}$ and $A x=\left(a_{k} x_{k}\right)$ for $x=\left(x_{k}\right) \in D(A)$. Then:

(i) $D(A)$ is not dense in $X$.

(ii) $A$ is not the generator of any exponentially Lipschitz continuous $(n+1)$-times integrated semigroup on $X$, for any nonnegative integer $n$.

(iii) $A$ is the generator of the locally Lipschitz continuous twice integrated semigroup $\{U(t) ; t \geq 0\}$ on $X$ defined by

$$
U(t) x=\left(\int_{0}^{t}(t-s) \exp \left(a_{k} s\right) x_{k} d s\right)
$$

for $x=\left(x_{k}\right) \in X$ and $t \geq 0$.

Proof. Since $\lim _{k \rightarrow \infty}\left|a_{k}\right|=\infty$ we have $\lim _{k \rightarrow \infty} x_{k}=0$ for $x=\left(x_{k}\right) \in$ $D(A)$, which implies (i). To prove (ii), assume to the contrary that $A$ is the generator of an exponentially Lipschitz continuous $(n+1)$-times integrated semigroup $\{S(t) ; t \geq 0\}$ on $X$ for some nonnegative integer $n$. By (2.2) we have $S(t) x=\left(\int_{0}^{t}\left((t-s)^{n} / n !\right) \exp \left(a_{k} s\right) x_{k} d s\right)$ for $x=\left(x_{k}\right) \in X$ and 
$t \geq 0$, since the $k$ th component $f_{k}(t)$ of $S(t) x$ must satisfy the equation $f_{k}(t)=\int_{0}^{t} a_{k} f_{k}(s) d s+\left(t^{n+1} /(n+1) !\right) x_{k}$ for $t \geq 0$.

Let $l \geq 1$. Then we have

$$
\left|a_{k}\right|^{-l}\left|\exp \left(a_{k} t\right)\right|=\exp (k(t-l k))\left(1+k^{2} e^{-2 k^{2}}\right)^{-l / 2}
$$

and $\sup _{k \geq 1} \exp (k(t-l k))=\exp \left(t^{2} / 4 l\right)$ for $t \geq 2 l$. Since

$$
\int_{0}^{t} \frac{(t-s)^{l-1}}{(l-1) !} \exp \left(a_{k} s\right) d s=\left(a_{k}\right)^{-l} \exp \left(a_{k} t\right)-\sum_{p=1}^{l}\left(a_{k}\right)^{-p} \frac{t^{l-p}}{(l-p) !}
$$

and $\left|\sum_{p=1}^{l}\left(a_{k}\right)^{-p} t^{l-p} /(l-p) !\right| \leq e^{t}$ for $t \geq 0$ and $k \geq 1$, there exist $C_{l} \geq$ $c_{l}>0$ such that

$$
c_{l} \exp \left(t^{2} / 4 l\right)-e^{t} \leq \sup _{k \geq 1}\left|\int_{0}^{t} \frac{(t-s)^{l-1}}{(l-1) !} \exp \left(a_{k} s\right) d s\right| \leq C_{l} \exp \left(t^{2} / 4 l\right)+e^{t}
$$

for $t \geq 2 l$, where the second inequality is true for all $t \geq 0$. By (3.4) with $l=n+1$ we see that $\|S(t)\|\left(=\sup _{k \geq 1}\left|\int_{0}^{t}\left((t-s)^{n} / n !\right) \exp \left(a_{k} s\right) d s\right|\right)$ is not exponentially bounded, which contradicts the fact that $\{S(t) ; t \geq 0\}$ is exponentially Lipschitz continuous.

Finally, we prove (iii). We use the inequality (3.4) with $l=1$ to obtain $\|U(t)-U(s)\| \leq\left(C_{1} e^{t^{2} / 4}+e^{t}\right)(t-s)$ for $t \geq s \geq 0$, which implies that $\{U(t) ; t \geq 0\}$ is a locally Lipschitz continuous family in $B(X)$. The functional equation (I2) with $n=2$ is clearly satisfied. If $B$ is the generator of $\{U(t) ; t \geq 0\}$ then it is obvious that $A \subset B$. Since $\varrho(A) \supset \mathbb{R}$, the intersection of $\varrho(A)$ and $\varrho(B)$ is nonempty. The above two facts together imply $A=B$.

REMARK 3.1. In [4], the relationship between integrated semigroups and regularized semigroups was investigated. In this direction, it is seen from the above proof that the following result holds: Let $A$ be a closed linear operator in $X$ with nonempty resolvent set $\varrho(A)$. Let $n$ be a nonnegative integer and $c \in \varrho(A)$. Then the following statements are mutually equivalent:

(i) $A$ is the generator of a locally Lipschitz continuous $(n+1)$-times integrated semigroup on $X$.

(ii) $A$ is the generator of a locally Lipschitz continuous $C$-regularized semigroup on $X$ with $C=(c I-A)^{-(n+1)}$.

(iii) The resolvent set of $A$ contains $(\omega, \infty)$ for some $\omega \geq 0$. For each $\tau>0$ there exists $M_{\tau}>0$ such that $\left\|\prod_{l=1}^{k}\left(I-h_{l} A\right)^{-1} x\right\| \leq M_{\tau}\|x\|_{n}$ for $x \in D\left(A^{n}\right)$ and every finite sequence $\left\{h_{l}\right\}_{l=1}^{k}$ of positive numbers such that $h_{l} \omega<1$ for $1 \leq l \leq k$ and $\sum_{l=1}^{k} h_{l} \leq \tau$. 
REMARK 3.2. In [9], the generators of integrated semigroups were characterized in terms of the associated abstract Cauchy problems. See also [3] and [4].

REMARK 3.3. Even for any local $(n+1)$-times integrated semigroup $\{U(t) ; t \in[0, T)\}$ which is locally Lipschitz continuous, the definition (1.1) of generators makes sense. However, we do not know whether the non-densely defined generators satisfy $(2.1)$ and $(2.2)$ for $t \in[0, T)$. Notice that a complex characterization of another type of "generators" was given in [2]. The problem of real characterization of the non-densely defined generators of such local integrated semigroups remains open except for our case of $T=\infty$, although a Hille-Yosida type theorem was found in [15, Theorem 4.2]. (See also [6].)

Acknowledgements. The author is greatly indebted to the referee for several helpful comments, and acknowledges support by the Grant-in-Aid for Scientific Research (C)(2) No. 16540153, Japan Society for the Promotion of Science.

\section{References}

[1] W. Arendt, Vector-valued Laplace transforms and Cauchy problems, Israel J. Math. 59 (1987), 327-352.

[2] W. Arendt, O. El-Mennaoui, V. Keyantuo, Local integrated semigroups: evolution with jumps of regularity, J. Math. Anal. Appl. 186 (1994), 572-595.

[3] W. Arendt, C. Batty, M. Hieber and F. Neubrander, Vector-Valued Laplace Transforms and Cauchy Problems, Monogr. Math. 96, Birkhäuser, Basel, 2001.

[4] R. deLaubenfels, Existence Families, Functional Calculi and Evolution Equations, Lecture Notes in Math. 1570, Springer, Berlin, 1994.

[5] H. Kellerman and M. Hieber, Integrated semigroups, J. Funct. Anal. 84 (1989), 160-180.

[6] V. Keyantuo, C. Müller and P. Vieten, The Hille-Yosida theorem for local convoluted semigroups, Proc. Edinburgh Math. Soc. 46 (2003), 395-413.

[7] Y. Kobayashi, Difference approximation of Cauchy problems for quasi-dissipative operators and generation of nonlinear semigroups, J. Math. Soc. Japan 27 (1975), 640-665.

[8] I. Miyadera, S. Oharu and N. Okazawa, Generation theorems of semi-groups of linear operators, Publ. Res. Inst. Math. Sci. 8 (1973), 509-555.

[9] I. Miyadera, M. Okubo and N. Tanaka, $\alpha$-times integrated semigroups and abstract Cauchy problems, Mem. School Sci. Engrg. Waseda Univ. 57 (1993), 267-289.

[10] F. Neubrander, Integrated semigroups and their applications to the abstract Cauchy problem, Pacific J. Math. 135 (1988), 111-155.

[11] S. Oharu, Semigroups of linear operators in a Banach space, Publ. Res. Inst. Math. Sci. 7 (1971/72), 205-260.

[12] N. Sanekata, Some remarks on the abstract Cauchy problem, ibid. 11 (1975), 51-65.

[13] N. Tanaka and I. Miyadera, Some remarks on C-semigroups and integrated semigroups, Proc. Japan Acad. Ser. A Math. Sci. 63 (1987), 139-142. 
[14] N. Tanaka and N. Okazawa, Local C-semigroups and local integrated semigroups, Proc. London Math. Soc. 61 (1990), 63-90.

[15] S. W. Wang, Hille-Yosida type theorems for local regularized semigroups and local integrated semigroups, Studia Math. 152 (2002), 45-67.

[16] K. Yosida, Functional Analysis, 6th ed., Springer, Berlin, 1980.

Department of Mathematics

Faculty of Science

Okayama University

Okayama 700-8530, Japan

E-mail: tanaka@math.okayama-u.ac.jp

Received March 7, 2003

Revised version October 19, 2004 